\title{
A Novel Routing Protocol for Realistic Traffic Network Scenarios in VANET
}

\author{
Gagan Deep Singh $\mathbb{D}^{1},{ }^{1}$ Sunil Kumar ${ }^{\mathbb{D}},{ }^{1}$ Hammam Alshazly $\mathbb{D}^{\mathbb{D}}{ }^{2}$ Sahar Ahmed Idris, ${ }^{3}$ \\ Madhushi Verma $\left(\mathbb{D},{ }^{4} \text { and Samih M. Mostafa }{ }^{2}\right)^{2}$ \\ ${ }^{1}$ University of Petroleum and Energy Studies, Dehradun-, 248007 Uttarakhand, India \\ ${ }^{2}$ Faculty of Computers and Information, South Valley University, Qena 83523, Egypt \\ ${ }^{3}$ College of Industrial Engineering, King Khalid University, Abha, Saudi Arabia \\ ${ }^{4}$ School of Engineering and Applied Sciences, Bennett University, Greater Noida, India
}

Correspondence should be addressed to Hammam Alshazly; hammam.alshazly@sci.svu.edu.eg

Received 10 October 2021; Accepted 11 November 2021; Published 9 December 2021

Academic Editor: Mohammed H. Alsharif

Copyright (c) 2021 Gagan Deep Singh et al. This is an open access article distributed under the Creative Commons Attribution License, which permits unrestricted use, distribution, and reproduction in any medium, provided the original work is properly cited.

\begin{abstract}
The vehicular ad hoc network (VANET) has traditional routing protocols that evolved from mobile ad hoc networks (MANET). The standard routing protocols of VANET are geocast, topology, broadcast, geographic, and cluster-based routing protocols. They have their limitations and are not suitable for all types of VANET traffic scenarios. Hence, metaheuristics algorithms like evolutionary, trajectory, nature-inspired, and ancient-inspired algorithms can be integrated with standard routing algorithms of VANET to achieve optimized routing performance results in desired VANET traffic scenarios. This paper proposes integrating genetic algorithm (GA) in ant colony optimization (ACO) technique (GAACO) for an optimized routing algorithm in three different realistic VANET network traffic scenarios. The paper compares the traditional VANET routing algorithm along with the metaheuristics approaches and also discusses the VANET simulation scenario for experimental purposes. The implementation of the proposed approach is tested on the open-source network and traffic simulation tools to verify the results. The three different traffic scenarios were deployed on Simulation of Urban Mobility (SUMO) and tested using NS3.2. After comparing them, the results were satisfactory and it is found that the GAACO algorithm has performed better in all three different traffic scenarios. The realistic traffic network scenarios are taken from Dehradun City with four performance metric parameters including the average throughput, packet delivery ratio, end-to-end delay, and packet loss in a network. The experimental results conclude that the proposed GAACO algorithm outperforms particle swarm intelligence (PSO), ACO, and Ad-hoc on Demand Distance Vector Routing (AODV) routing protocols with an average significant value of $1.55 \%, 1.45 \%$, and $1.23 \%$ in three different VANET network scenarios.
\end{abstract}

\section{Introduction}

VANETs have evolved as a key solution of intelligent transport systems (ITS). The existing technologies and swarm intelligence are also integrating with VANET to realize its actual purpose. VANET routing protocols are evolved from the standard preexisting protocols like Dynamic Source Routing (DSR) and AODV. DSR and AODV were found efficient and best suitable for multihop wireless ad hoc networks and Internet of Things (IoT) devices [1]. While designing any of the VANET, scenarios for Vehicle-to-
Vehicle (V2V) communication must focus on all the aspects of VANET routing. These days, the technological upgrades are very frequent and fast. Hence, soon, it will be noticed that the deployment of the 5G GSM network invades in Vehicle-to-Infrastructure (V2I) setup. That results in the inception of V2V and V2I in the Urban Intelligent Transportation Systems (UITS). The emergence of IoT in near future will boost VANET communication through machine learning and data analysis. VANET is made through vehicles independently communicating among themselves. These vehicles can be assumed as nodes capable enough to 


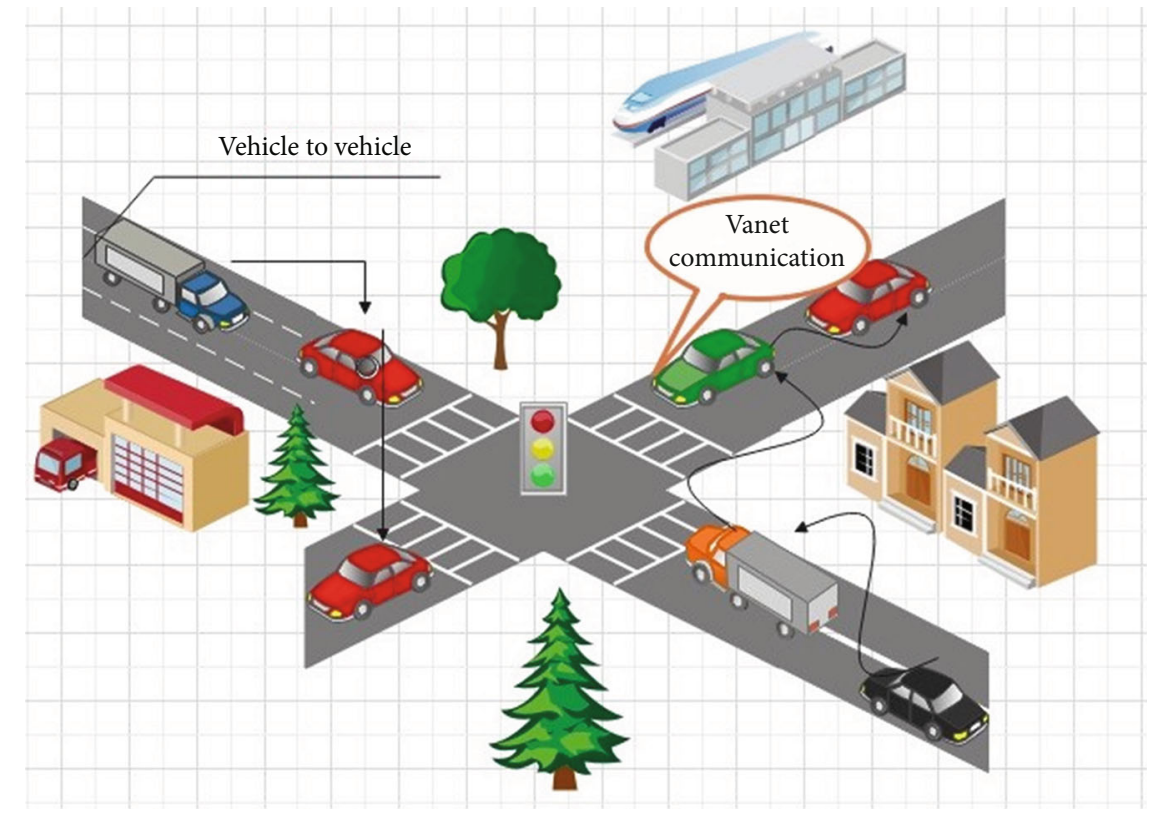

Figure 1: VANET structure.

establish wireless communication with other nodes, giving birth to entirely a new shared mesh network with the selforganizing property. It generates huge possibilities to develop numerous applications for VANET. These applications can make the road travel experience easy, safe, more entertaining, and efficient. It will also help decrease the traveling time; road traffic congestion helps avoid congested areas, increase road capacity, and be usable during emergencies, thus, resulting in lesser fuel wastage [2]. Ultimately, the environment will be cleaner. At present, various VANET routing protocols and wireless standards are already available, but none of them is yet able to provide the universal routing solution for VANET realistic scenarios [3].

Figure 1 presents the VANET structure showing how the vehicle is passing a broadcast message to other vehicle nodes and creates a VANET environment. In this dynamic structure, vehicles communicate with each other to broadcast the information. The routing plays an integral role in this structure, and different routing challenges are faced in this type of vehicular networks such as dynamic topology, availability and reliability of network throughput, realistic traffic scenarios, unpredictable driver's driving psychology, and inadequate routing algorithm for all types of vehicular traffic scenarios. The research paper addresses the identified challenges and provides an effective routing mechanism focusing on performance metrics like average throughput, packet delivery ratio, and end-to-end delay in VANET routing. follows:

The major contributions of this research paper are as

(i) A novel genetic algorithm-based ant colony optimization technique (GAACO) was proposed to optimize the routing algorithm for three realistic traffic scenarios (ii) The proposed algorithm validated the VANET routing performance with average throughput, packet delivery ratio, end-to-end delay, and packet loss

(iii) The performance of the proposed GAACO is discussed and compared with the traditional protocols using the NS3.26 simulator with standard and realistic traffic scenarios

The rest of the paper is structured as follows. The various research issues of VANETs with the significance of routing in VANETs are reviewed in Section 2. The methodology adopted along with the designed framework is discussed in Section 3. The experimental setup and results are discussed in Section 4. Section 5 draws the conclusion of this research work.

\section{Overview of Previous Work}

The routing protocols are responsible for providing the best suitable route among the nodes within its network. There are some standard routing protocols designed for VANET routing environments. These protocols are classified in various aspects, like quality of service (QoS), characteristics of the protocol, network structure, routing algorithm, and information dissemination [1]. The literature review of this paper discussed five major VANET routing protocols, which are presented in Figure 2.

Further, in the literature review, the routing protocols in VANET are classified into transmission strategies-based routing protocols and information-based routing protocols [4]. It is concluded during rigorous literature finding that routing information-based protocol is suitable for this research development, as this needs to devise efficient routing based on swarm intelligence. It is also confirmed 


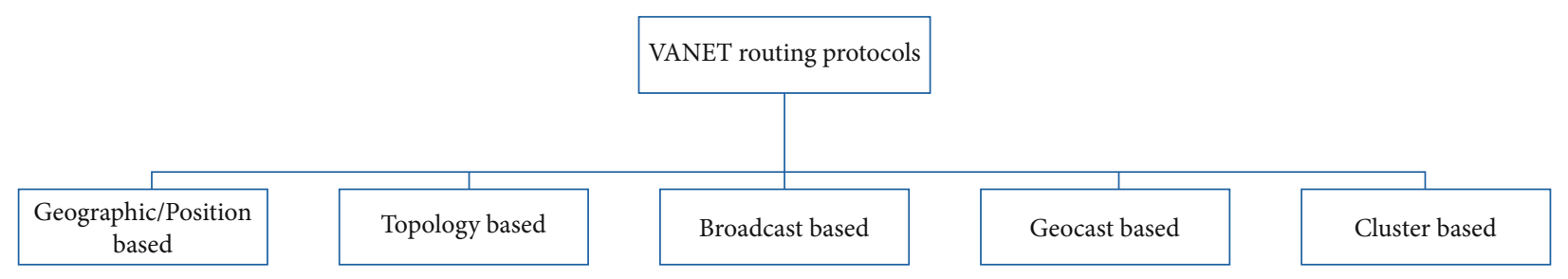

FIGURE 2: Classification of VANET routing protocols.

that the literatures are not concerned with transmission strategy-based protocol, as the research is not focusing on information dissemination issues and challenges in VANET [5].

Hence, this extensive literature survey can conclude that existing standard routing protocols are not suitable for VANET routing in all traffic scenarios. The informationbased routing protocol can be classified into two classes, i.e., topology-based and geographic/position-based VANET routing protocols. Every node is aware of the network layout in topology-based routing, whereas each node knows the location of other nodes while forwarding the packet in position-based routing [6]. It was seen that VANETs are not scale-free networks as Gaussian probability circulations approximate their node degree distributions. However, the conduct of low-density VANETs is the same as to smallworld networks, and connectivity is too low even to consider benefitting from the small-world property [7].

In [8], a navigation methodology that can gather online information of the roads through VANET can be used by the drivers to reach their destination in a distributed manner and real-time situation. The simulation test result shows that the new protocol increases the vehicle's rate of local awareness through simulated barriers. Exchanged messages updated the neighbouring vehicle's records and increased attention to different nodes that cooperatively forwarded demands and solutions. Intervehicular interaction that assessed another independent WiMAX framework is proposed in [9]. The broad simulation was acknowledged in the OPNET framework for the design of the WiMAXmesh framework. In [3], the authors presented their platooning algorithm based on swarm-based intelligence. This algorithm applied two different factors to decrease the travel time. It insisted on green traffic signal time that makes the traffic-free path for the vehicle movement. Then, the proposed preemptive traffic signal approach is combined with an existing modified ACO technique to design platooning of the vehicles. These characteristics concluded the platooning algorithm is an efficient way to minimize the waiting time of the commuters. In [10], the authors introduced an investigation of different simulation instruments accessible for VANET. VANET simulators' taxonomy assists future VANET analysts to pick an ideal simulator, which is most appropriate for VANET scheme objectives. Two AODV protocols, P-AODV and improved AODV, are examined and dependent on some parameters [11].

The available routing protocols are unable to facilitate VANET with efficient routing in major traffic scenarios [1]. The Optimized Link State Routing Protocol (OLSR) is

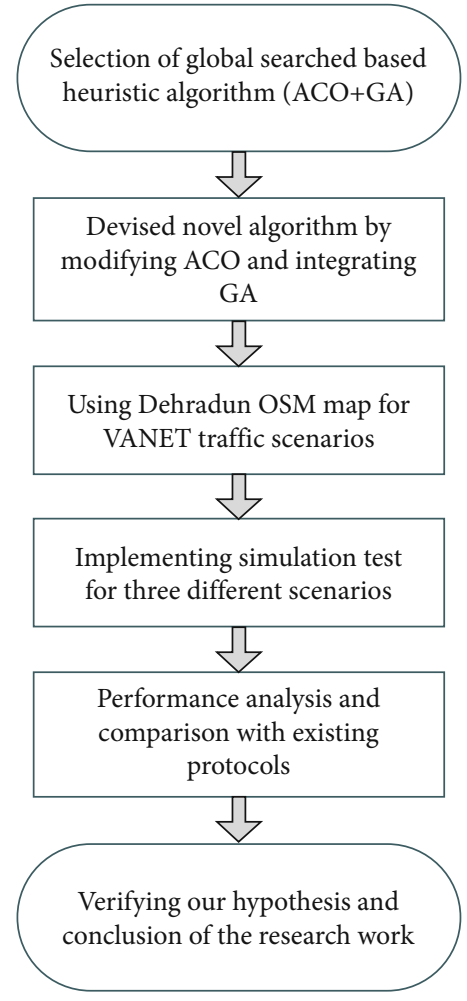

Figure 3: The adopted methodology.

not preferred as it requires high bandwidth to process and to identify the best network route [12]. In [13], GA is applied to manage the multicast routing through computational intelligence. A stable routing protocol resolves the node disconnection in VANET that was tested and alerted the disconnection before occurrence [14]. The multiconstrained QoS aware routing algorithm is also developed using ACO-based swarm intelligence techniques. As a result, the traffic type data-based QoS network can be achieved. So, security in VANET is also attained through a reliable QoS algorithm [15]. In VANET, the ACO and DSR protocols have also been proposed as routing options that use stable routing in a variety of VANET scenarios [16]. To monitor real-time performance, an eco-friendly ACO-based routing method was presented that shares roadway linkages. The novel feedback and cost updating technique are used to resolve the weakness of VANET routing [17]. In [18], the authors proposed Prediction-based Greedy Perimeter Stateless Routing (PGPSR), which is a modified version of Greedy Parameter Stateless Routing (GPSR). PGPSR is more efficient than the standard GPSR because packet delivery ratio 


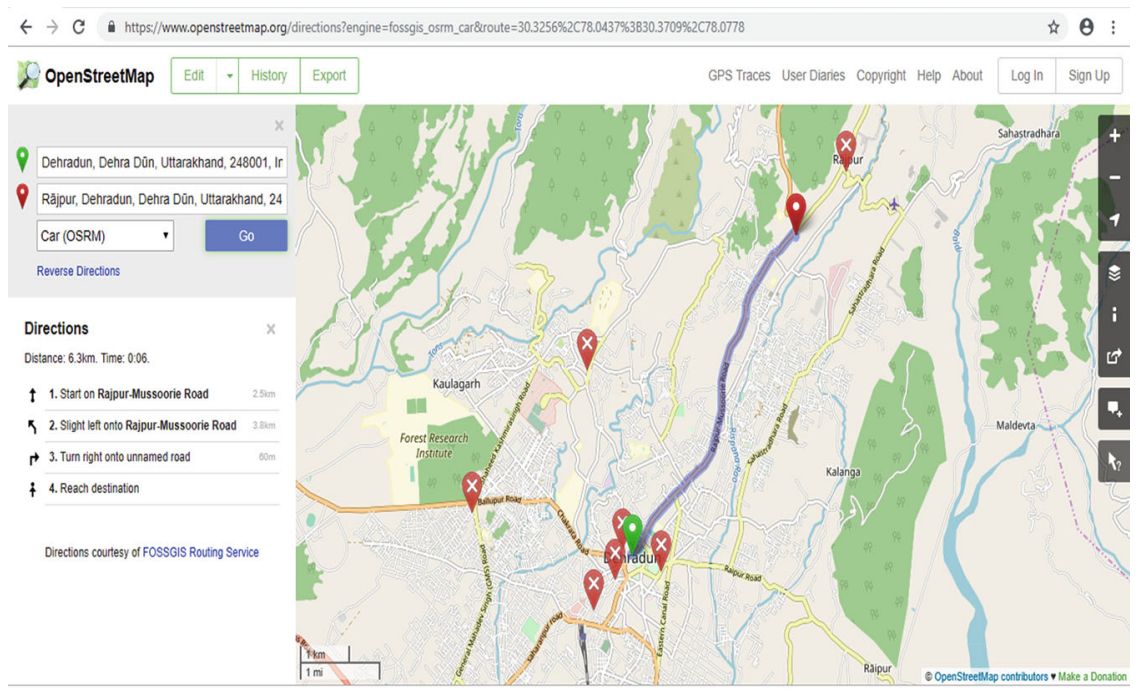

FIgure 4: OSM map of Dehradun Clock Tower to Mussoorie Rajpur Road.

(PDR) and maximum throughput are found to be increased in VANET routing. However, it does not fit dense and sparse network scenarios due to the significant delay in calculations and its complex computations. In [19], the authors proposed the GA technique for VANET to locate the best population of vehicles that generated and managed data flow and reduce the bandwidth utilization in wireless network. Using random values of the initial population size is reduced based on corresponding effect coefficients.

In [20], both PSO and ACO are considered in the vehicular network for enhancing the performance of VANET. Multiple routes from the source to the destination are established with uniform density of the vehicles using ACO. In [21], a new approach has been proposed and compared with the existing algorithms. The proposed algorithm showed improved performance in terms of delay time reduction, throughput, stability up gradation, and lifetime.

The improved genetic algorithm-based routing optimization technique (IGAROT) is presented in [22]. This GA variant replaces the selection technique with the $k$-means clustering method adopting from the same concept of a novel clustering-based genetic algorithm for the route optimization technique proposed in another approach [23]. IGAROT utilizes the vehicle density required in communication for VANET scenarios that randomly initialize individual populations. That provides the initial solution for the defined search space. Likewise, in a low-density VANET communication scenario with 20 vehicles, this makes a randomly generated initial population size of 1 by 20 . In this way, IGAROT creates unique generations by selecting the best solutions from the initial population. That comes after many generations to give the best solution. A new metaheuristic Giza pyramid construction (GPC) algorithm is presented in [24]. It helped to review the technologies, best optimum methods, and strategies of that time.

VANETs are unable to meet the exact needs and applications of all users. For example, in real-time situations, the emergency signals have to be forwarded with minimum latency and high priority; however, messages like infotainment and hello/hi can be put up in queue and latency. Hence, in sparse and dense networks, the minimum calculated desired time (MCDT) technique is suggested, and data dissemination is performed using a context-aware congestion resolution protocol. MCDT determines the node connectivity through a peak-stable link $[25,26]$. Modified lion algorithm (LA) is also used to compare with GA, and performance analysis was done for cost, complexity, and convergence. The simulation analysis of modified LA with respect to standard GA and LA proved the superiority of the modified LA [27]. In this literature study, the analysis of the related works in the last ten years was presented and then reviewed. Through that, the research approach is formed for efficient routing in VANET. The experimental test is performed on a realistic simulation environment using only open-source software tools like network simulator (NS) and SUMO.

\section{The Developed Methodology}

Simulations are used to test the performance of traditional and newly developed VANET routing algorithms. Many of them are suitable for the desired traffic scenario. But none of them can provide suitable results for three distinctive traffic scenarios. ACO technique with GA approach is selected and deployed for testing the performance in the selected scenarios along with two other traditional routing protocols of VANET. Then, simple traffic scenarios and complex traffic scenarios are used to generate the simulation environment. For a realistic traffic network scenario, the Dehradun City map of Clock Tower to Mussoorie-Rajpur road is imported from http://www.openstreemap.org [28]. The stepwise approach is adapted to complete this proposed research work as shown in Figure 3.

Then, a new algorithm is devised as per requirement analysis, by changing the position and updating the preexisting ACO by mutation features of GA. Hence, a new GAACO 


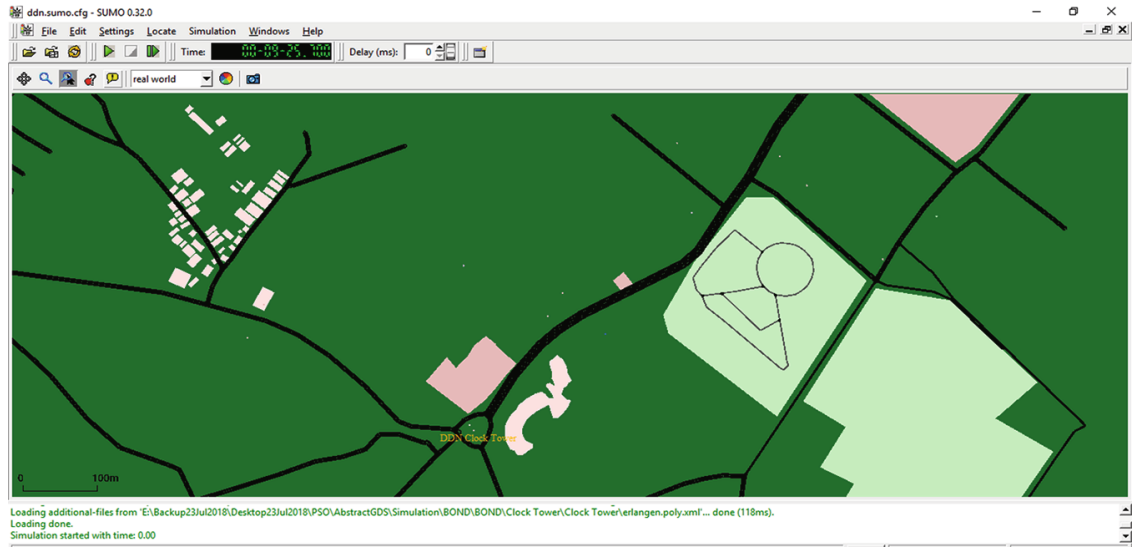

Figure 5: SUMO map generated through netconvert command for Dehradun Clock Tower to Mussoorie Rajpur Road.

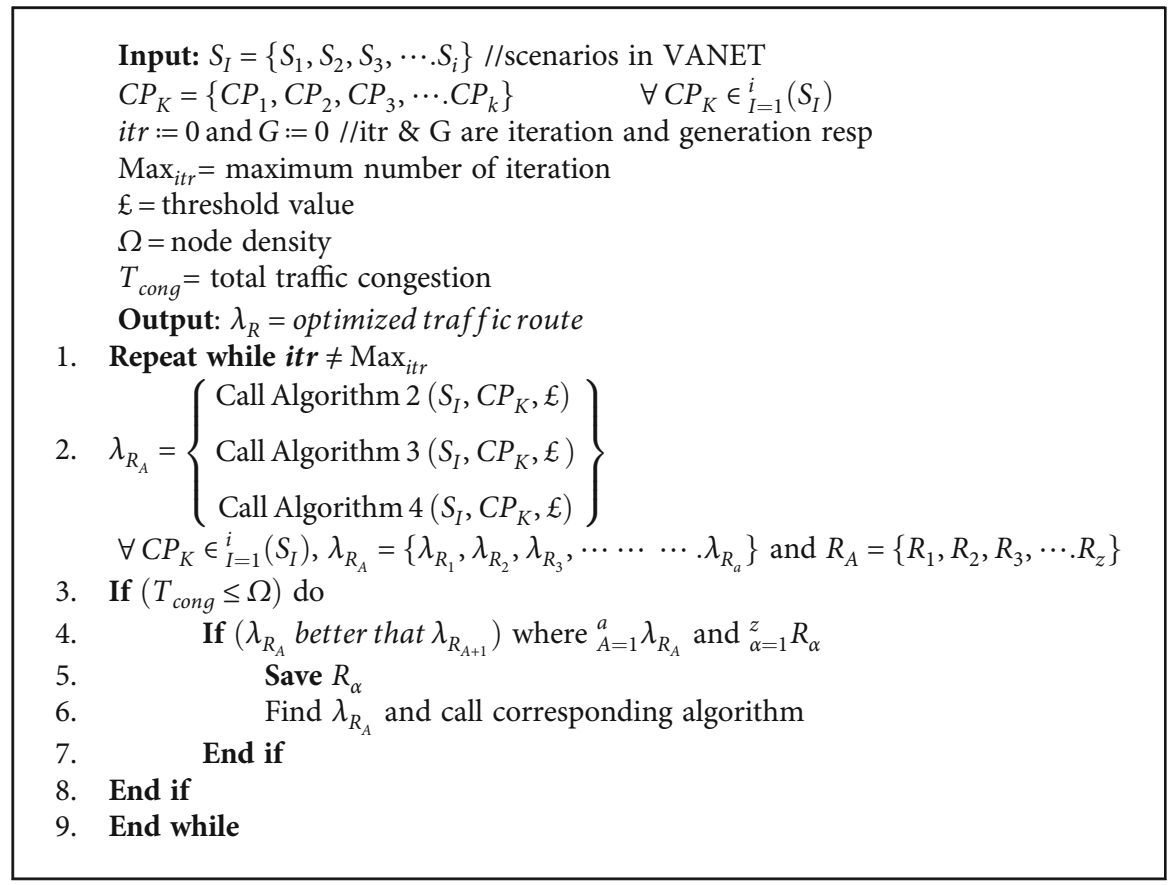

Algorithm 1: Optimized result based on the output of GA and ACO evaluation.

algorithm is proposed by rearranging the ACO population and integrating it with the GA algorithm. The experimental result shows the positive results of the algorithm on "Dehradun Clock Tower" to "Mussoorie-Rajpur Road" city route imported from http://openstreetmap.org [28]. The screenshots of the OpenStreamMap (OSM) map and generated route map are shown in Figures 4 and 5, respectively.

The selection of the population, crossover, and mutation is used for the VANET routing with ACO. In each iteration, a new population of the same size can be generated from the current population using three basic operations on the individuals of the population. GA is best suited for ACO techniques with road traffic scenarios in VANET as it is capable to adapt the routing alterations as per the need of the traffic scenarios.
The proposed algorithm is verified by validating the simulation results for three different traffic scenarios [3]. The algorithm devised for the research work is presented in Algorithm 1.

Further, the devised Algorithm 1 was deployed and tested using simulation experiments for all the mentioned algorithms as in Algorithm 2 to Algorithm 5. The methodology applied for simulation tests is elaborated as per Algorithm 1. The three different scenarios were designed in a simulation environment. The simple traffic network, complex traffic network, and Dehradun realistic city traffic network scenarios are implemented and tested to capture the routing performance data.

Algorithm 2 presents the deployment of PSO at three proposed traffic scenarios. This algorithm is used to run the simulation for gathering the data of the defined 


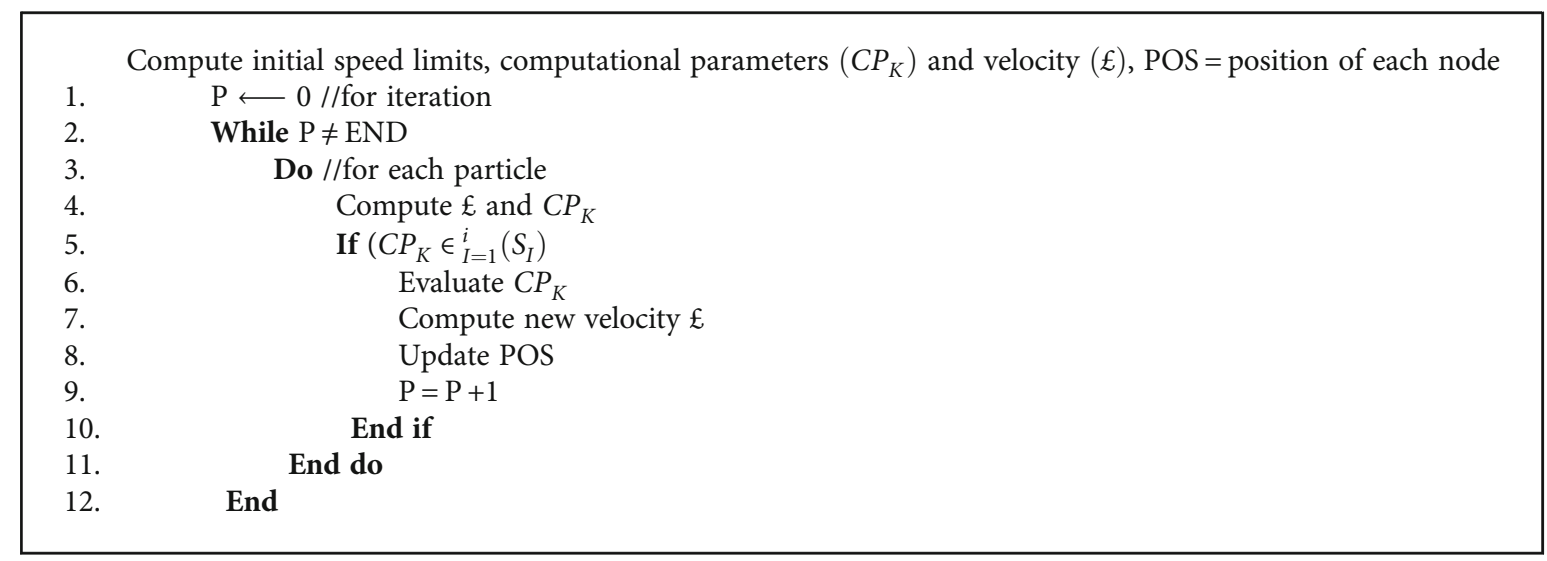

Algorithm 2: $\operatorname{PSO}\left(S_{I}, C P_{K}, £\right)$.

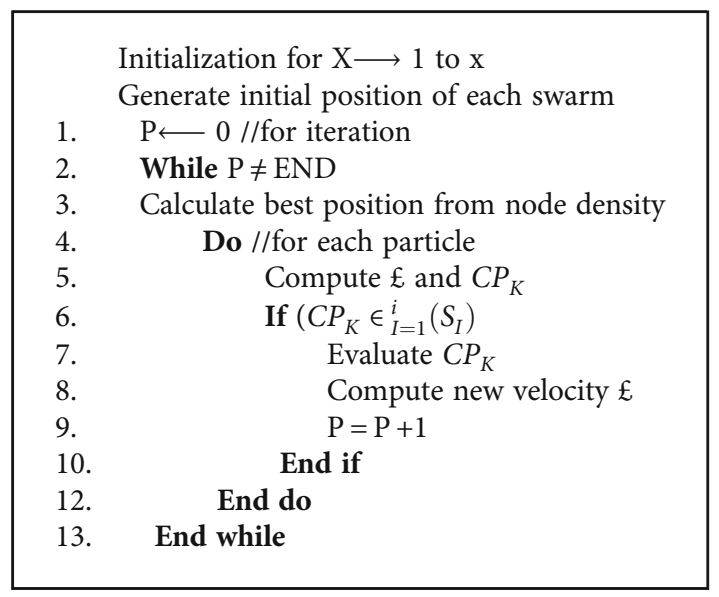

Algorithm 3: $\operatorname{ACO}\left(S_{I}, C P_{K}, £\right)$.

performance metrics. It is tested for average throughput, packet delivery ratio, end-to-end delay, and packet loss. All the values are tabulated for three proposed traffic scenarios for further analysis in comparison to ACO and devised GAACO algorithms.

Similarly, Algorithm 3 presented here is ACO technique that best suits the three proposed traffic scenarios. ACO is also deployed to run the simulation on these three traffic scenarios and to record the data of the opted performance metrics. The same method is applied to test for average throughput, packet delivery ratio, end-to-end delay, and packet loss. All the values are tabulated in comparison with PSO and devised GAACO algorithms.

Algorithm 4 presents our devised algorithm in a combination of ACO along with GA features. ACO is applied and has been tweaked by integrating the GA approach through Algorithm 5.

Algorithm 4 works on the basic features of ACO optimizing techniques taken vehicle nodes as ants in the swarm. Then, the population is updated as and when required through the mutation feature of the GA approach as illustrated in Algorithm 5. Algorithm 4 calls Algorithm 5 to regenerate the vehicle nodes for the simulation. Through this, the updated population of the vehicle swarm can be

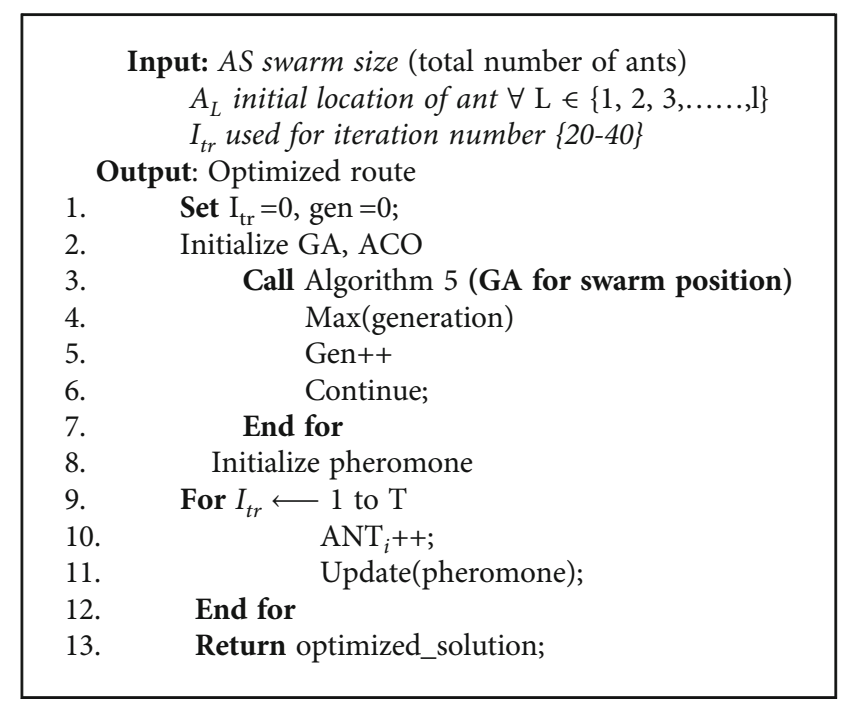

Algorithm 4: Proposed GAACO for optimizing parameter.

generated in the proposed traffic scenarios which is very beneficial in a realistic city traffic network environment.

Similarly, Algorithm 5 is also used to deploy the simulation test for the proposed traffic scenarios and evaluate the results. Hence, the optimized result is captured for further analysis and conclusion. The four selected performance metrics are used for all of the three scenarios during the implementation of PSO, ACO, and GAACO. The next part of the paper presents the framework designed to perform the simulation experiments and discussions of the results with the final analysis.

\section{Experiments and Results}

The methodology developed and applied for this research test provides a much simpler and faster way for performing experiments and getting results. For all the experimental setups, the test deployed on the computing machine with Corei7-8700 $3.2 \mathrm{GHz}$ of processor and a minimum of 16 GB RAM is required. That was the HP workstation used from the University of Petroleum and Energy Studies, 


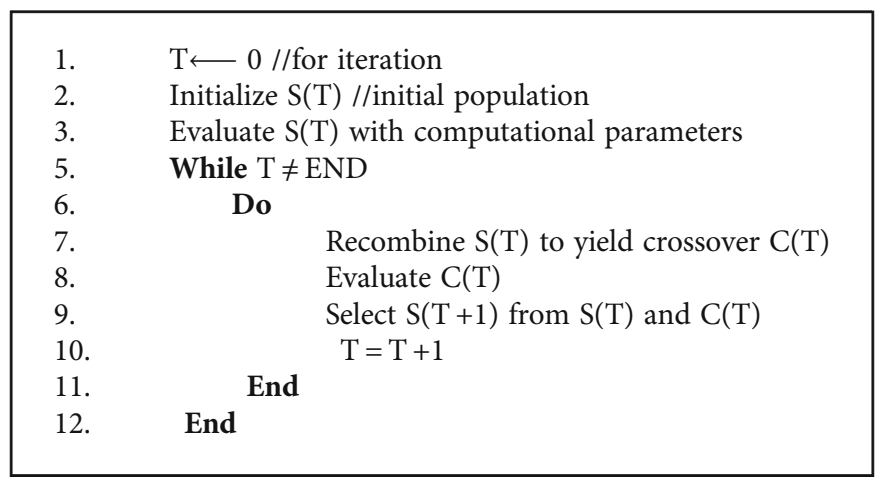

Algorithm 5: GA for initial and updated swarm position.

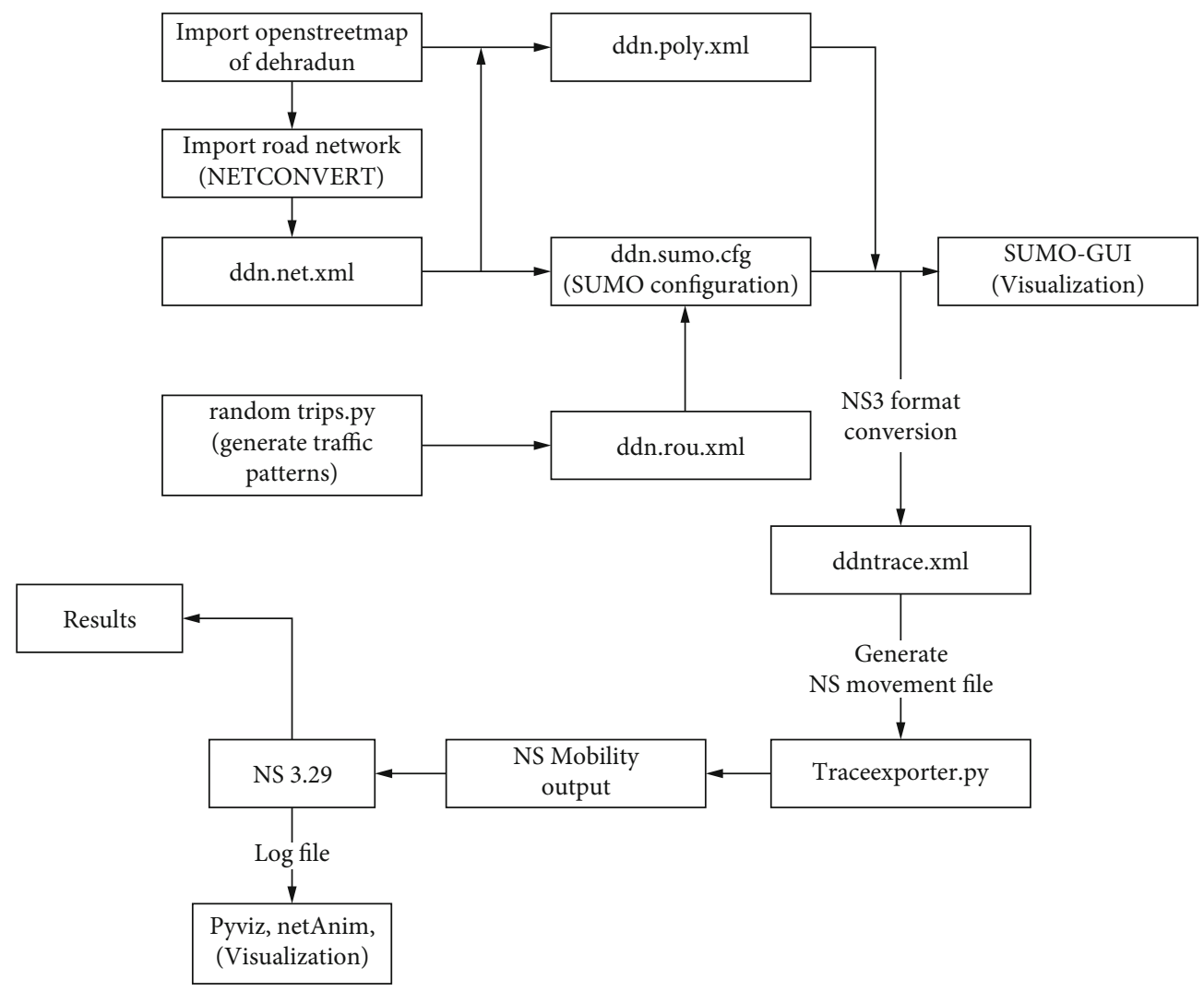

FIgURE 6: Framework used for simulation experiments.

Computer Laboratory, IT Tower, ITLab102_PC35. There was a need to repeat the simulation tests multiple times to verify the results, as reading may vary due to the machine's poor performance. Figure 6 illustrates the framework of the process used for all the research simulation tests. The below-designed framework was implemented for repetitive simulation tests on an HP workstation machine through the open-source operating system Ubuntu 16.04 release [29]. The traffic simulator used is SUMO 0.32 [30] and Network Simulator NS-3.26 $[31,32]$ for all the research tests.

The simulations were carried by importing the real city scenario map of Dehradun City from OpenStreetMap [33] for a realistic approach. Then, this was converted for the SUMO network. The region from Clock Tower to Mussoorie Rajpur Road is selected as it is the most congested route because of the heavy traffic going to Mussoorie from various regions during peak season times. The mentioned details in Table 1 illustrate the characteristics of the simulation parameters.

This paper presents the results from the point of view of four significant performance metrics, i.e., average throughput, packet delivery ratio, end-to-end delay, and packet loss. The simulation was performed for three VANET scenarios. The first is for simple, the second is for complex traffic networks, and the third is for real city Dehradun traffic 
TABLE 1: Simulation parameters with specifications.

\begin{tabular}{lc}
\hline Parameters & Specification details \\
\hline Open source OS & Ubuntu 16.04 \\
Open source network simulator & NS3.26 \\
Open source traffic simulator & Simulation of urban mobility (SUMO-0.32) \\
Open street map for Dehradun City vehicle traces & http://www.openstreetmap.org \\
Model & Manhattan mobility model \\
Transmission network range & 150 to $200 \mathrm{~m}$ \\
Size of data packets & 200 bytes \\
Interval & 0.02 seconds \\
Data rate & 2 Mbps \\
Protocol & MAC layer $802.11 \mathrm{p}$ \\
Velocity & 20 to $80 \mathrm{~km} / \mathrm{h}$ (above 80 is not permitted in Dehradun) \\
\hline
\end{tabular}

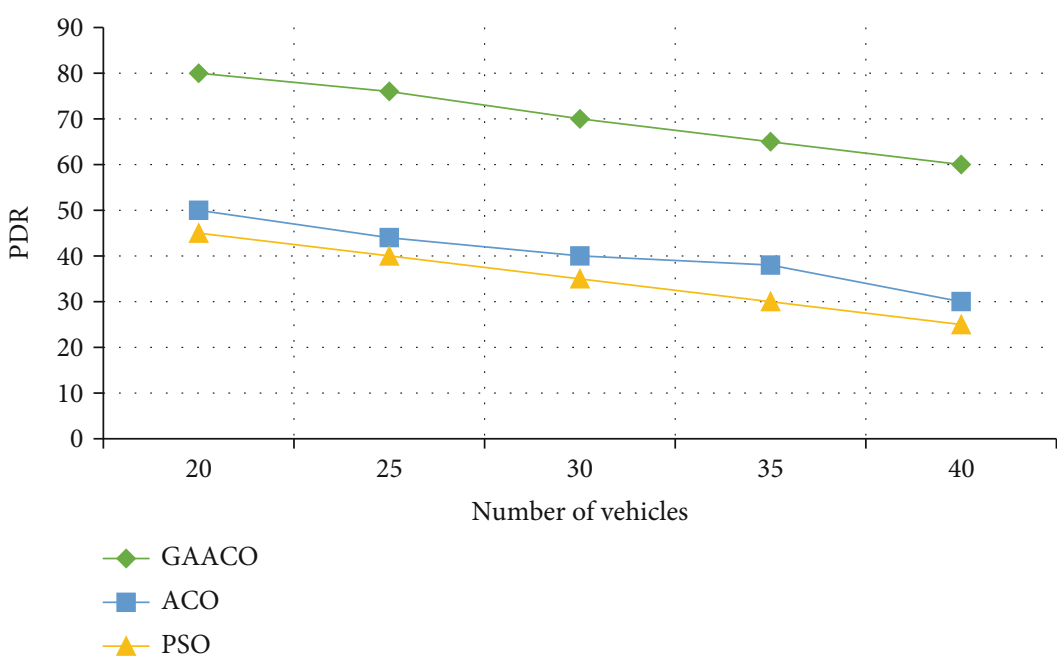

FIGURE 7: Performance analysis of packet delivery ratio computed for simple traffic network at a random speed.

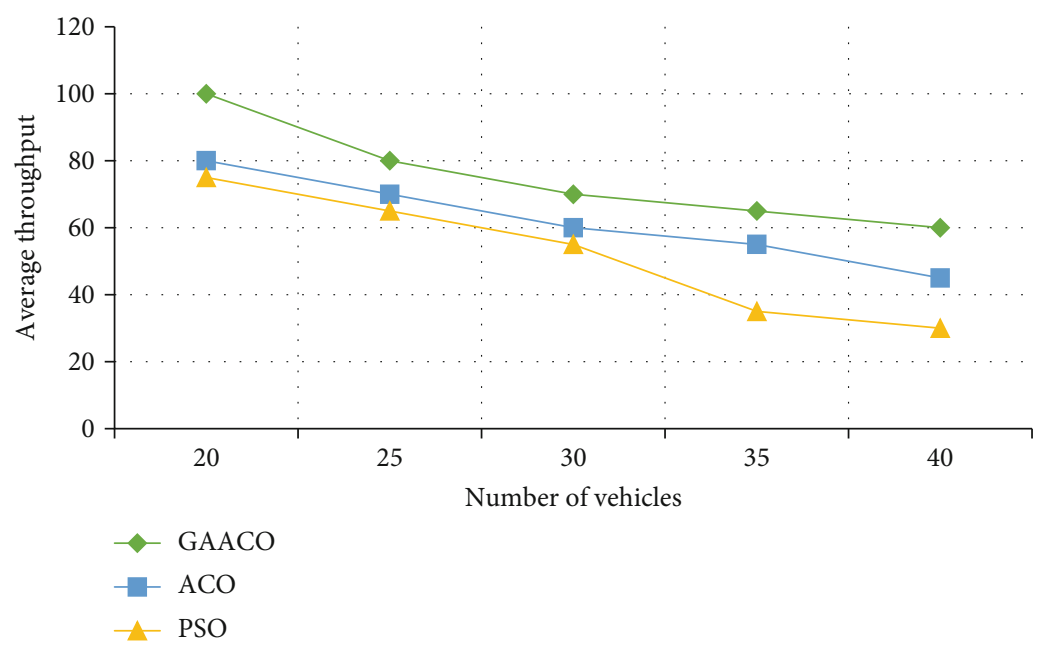

FIgURe 8: Performance analysis of average throughput computed for simple traffic network at a random speed.

scenarios. The above-discussed methodology is followed and tested for PSO, ACO, AODV, and GAACO routing protocols. The performance analysis is shown in graphs as per Figures 7-12.
In addition to the above-depicted comparison, the performance of the proposed GAACO is also compared with $\mathrm{ACO}, \mathrm{PSO}$, and AODV routing protocols from the point of view of end-to-end delay and packet loss. Figures 13 


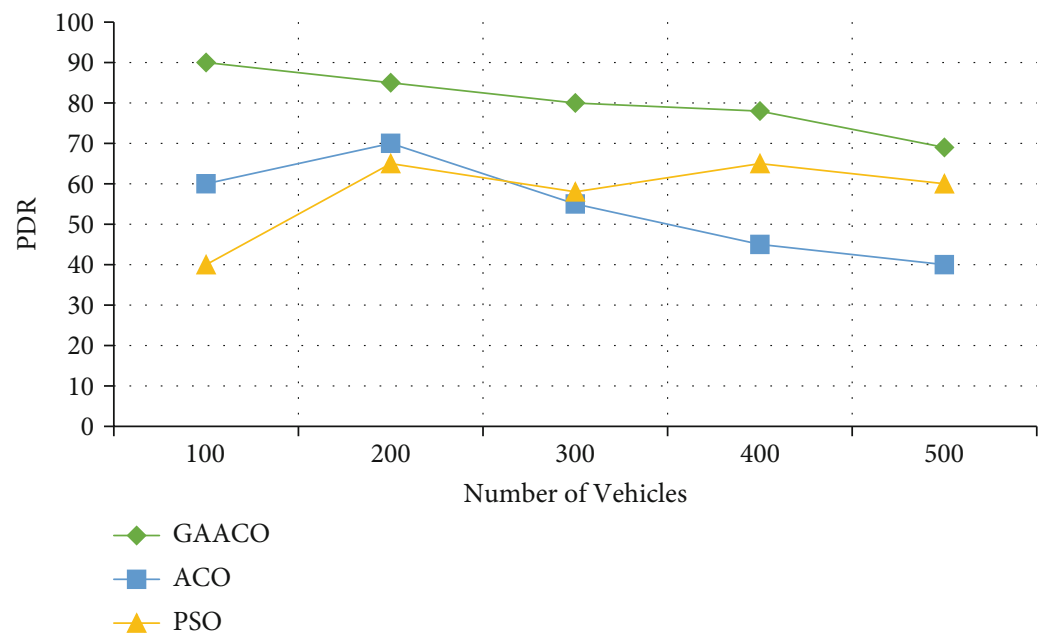

Figure 9: Performance analysis of packet delivery ratio computed for complex traffic network at a random speed.

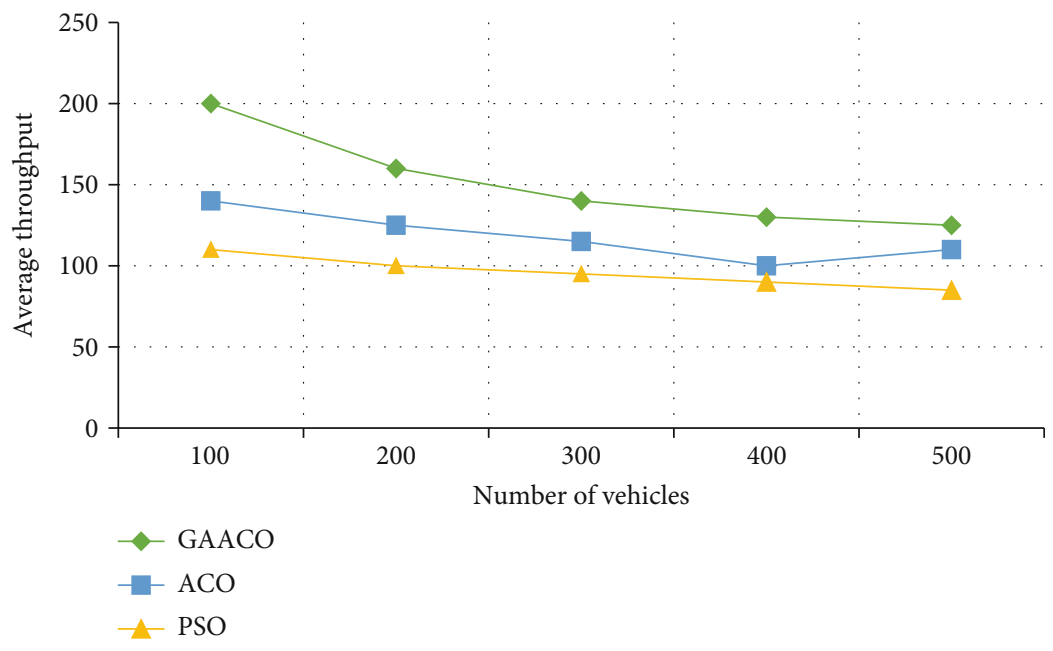

Figure 10: Performance analysis of average throughput w.r.t. no. of vehicles for dense network.

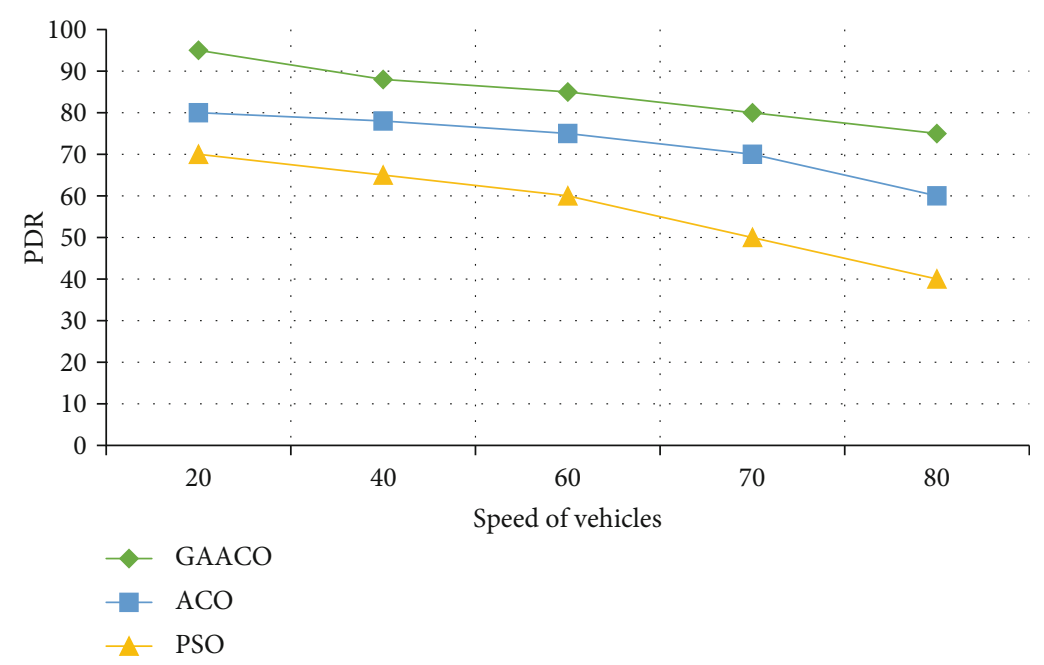

FIGURE 11: Performance analysis of packet delivery ratio computed for Dehradun realistic traffic scenario. 


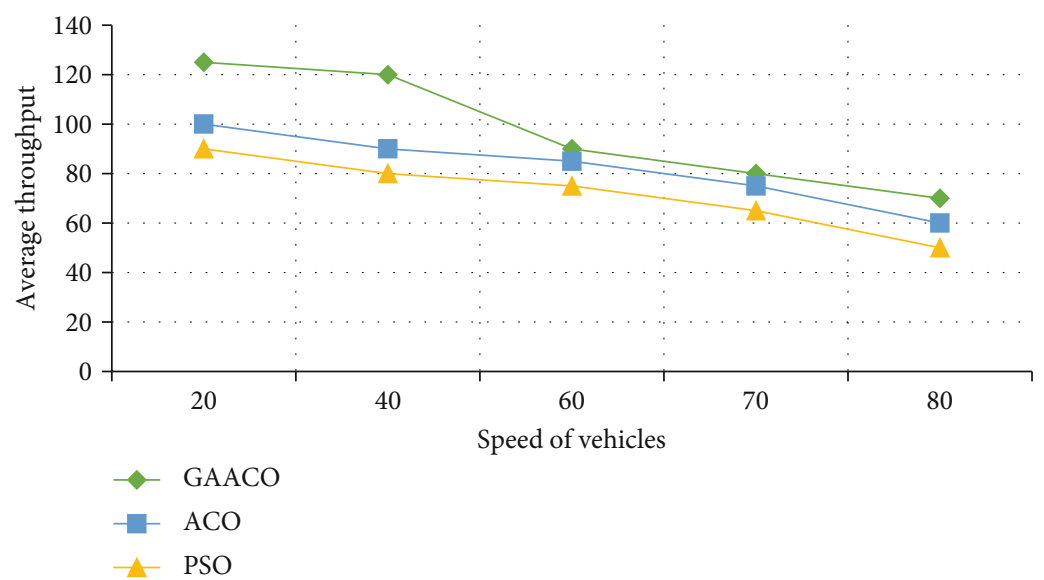

FIgURE 12: Performance analysis average throughput computed for Dehradun realistic traffic scenario.

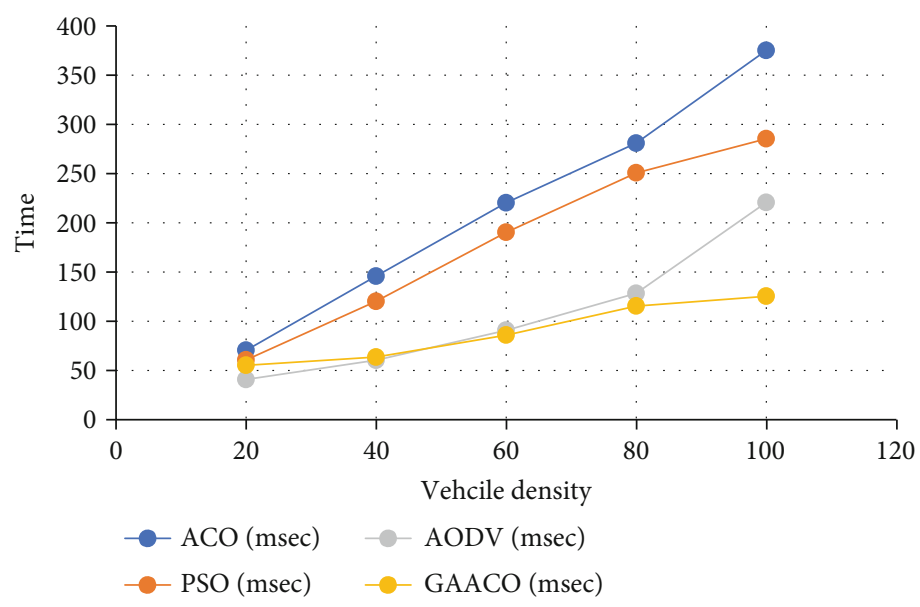

Figure 13: Performance analysis for end-to-end delay.

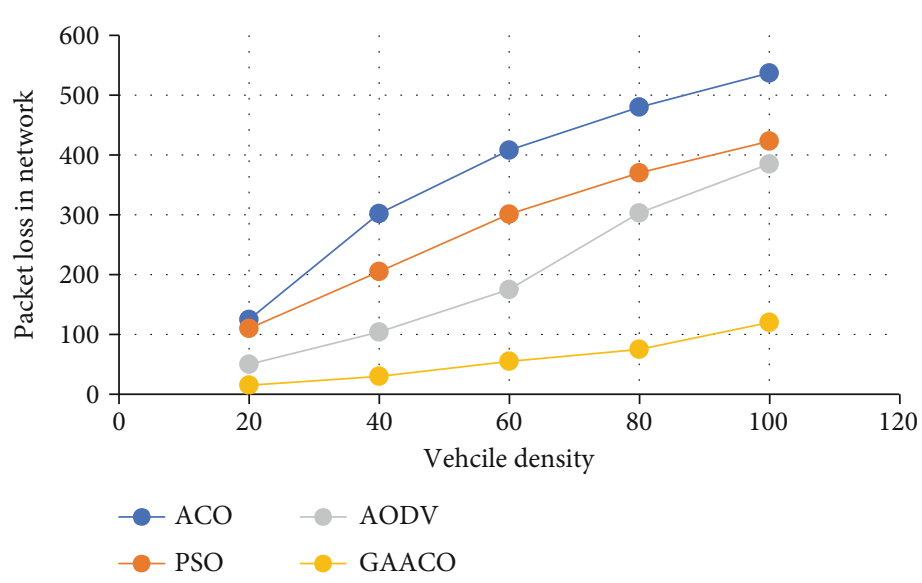

FIGURE 14: Performance analysis for packet loss in the network.

and 14 show that the proposed algorithm surpassed the compared algorithms in the selected VANET traffic scenarios.

Table 2 shows that the GAACO has better performance for two standard performance metrics in three distinctive
VANET traffic network scenarios, i.e., simple traffic, complex traffic, and Dehradun Mussoorie-Rajpur road realistic traffic network scenarios. The performance significance statistics are shown in comparison with PSO and ACO with respect to GAACO in Table 2. 
TABLE 2: Performance comparison with existing approaches.

\begin{tabular}{lccc}
\hline Routing algorithm & $\begin{array}{c}\text { Performance significance comparison } \\
\text { Simple traffic } \\
\text { network }\end{array}$ & $\begin{array}{c}\text { Complex traffic } \\
\text { network }\end{array}$ & $\begin{array}{c}\text { Dehradun realistic traffic } \\
\text { scenario }\end{array}$ \\
\hline $\begin{array}{l}\text { PDR performance significance of GAACO with PSO } \\
\text { PDR performance significance of GAACO with ACO }\end{array}$ & $1.9 \%$ & $1.4 \%$ & $1.5 \%$ \\
$\begin{array}{l}\text { Average throughput performance significance of GAACO } \\
\text { with PSO }\end{array}$ & $1.4 \%$ & $1.6 \%$ & $1.1 \%$ \\
$\begin{array}{l}\text { Average throughput performance significance of GAACO } \\
\text { with ACO }\end{array}$ & $1.2 \%$ & $1.3 \%$ & $1.3 \%$ \\
\hline
\end{tabular}

\section{Conclusion}

In this paper, the discussion on various research areas of VANET was presented, and a detailed review has been done on various traditional and metaheuristics VANET algorithms. Considerable amount of work has been done in the field of VANET routing, but such works are limited to specific scenarios and routing. They are also not able to fulfill the present challenges in various traffic routing scenarios. There is a huge scope to develop and test much better efficient routing that can work in multiple traffic scenarios. The present work has been done to fulfill the gap that has been presented through research challenges in VANET. Some techniques proved better in sparse networks while others in dense networks. Similarly, no such technique exists in the literature that can provide a solution to VANET routing for realistic traffic scenarios with a hybrid algorithm that incorporates swarm intelligence and genetic algorithm. The newly devised GAACO algorithm is compared on three different VANET traffic scenarios, and it is found that GAACO has performed better for three distinctive VANET network traffic scenarios. The significance of GAACO is found to be superior with an average significant value of $1.55 \%$, $1.45 \%$, and $1.23 \%$ for PSO, ACO, and AODV routing protocols with respect to simple traffic, complex traffic, and Dehradun realistic VANET traffic scenarios, respectively. Hence, this can be confidently concluded that the newly devised GAACO has the features that are best suited for considered VANET traffic routing environments. The routing performance is noticeably improved when it is tested for packet delivery ratio and average throughput. Moreover, there were improvements in end-to-end delay and packet loss in a network. The future scope of this research can be considered to test and implement the proposed algorithm in other VANET scenarios with different performance metrics. The same can also be tested in flying ad hoc network (FANET) and intrasatellite communications for space research programs.

\section{Data Availability}

No external data was used to support the conducted experiments.

\section{Conflicts of Interest}

The authors declare that they have no conflict of interest.

\section{Acknowledgments}

The authors extend their appreciation to the Deanship of Scientific Research at King Khalid University through the Research Groups Program under Grant RGP.2/53/42.

\section{References}

[1] G. D. Singh, R. Tomar, H. G. Sastry, and M. Prateek, "A review on VANET routing protocols and wireless standards," in Smart Innovation, vol. 78 of Systems and Technologies, pp. 329-340, Springer, Singapore, 2018.

[2] G. D. Singh, M. Prateek, and G. Hanumat Sastry, "Swarm intelligence based algorithm for efficient routing in VANET," International Journal of Innovative Technology and Exploring Engineering, vol. 9, no. 5, pp. 1124-1136, 2020.

[3] G. D. Singh, M. Prateek, and G. Hanumat Sastry, "Swarm intelligence based efficient routing algorithm for platooning in VANET through ant colony optimization," International Journal of Innovative Technology and Exploring Engineering, vol. 8, no. 9, pp. 1238-1244, 2019.

[4] S. Singh and G. S. Aujla, "A closer look through routing protocols in vehicular ad hoc networks (VANETs)," IOSR Journal of Engineering, vol. 4, no. 6, pp. 58-64, 2014.

[5] M. Chaqfeh, A. Lakas, and I. Jawhar, "A survey on data dissemination in vehicular ad hoc networks," Vehicular Communications, vol. 1, no. 4, pp. 214-225, 2014.

[6] C. Ksouri, I. Jemili, M. Mosbah, and A. Belghith, "VANETs routing protocols survey: classifications, optimization methods and new trends," in International Workshop on Distributed Computing for Emerging Smart Networks, pp. 3-22, Springer, Cham, 2019.

[7] J. Bhatia, R. Dave, H. Bhayani, S. Tanwar, and A. Nayyar, "SDN-based real-time urban traffic analysis in VANET environment," Computer Communications, vol. 149, pp. 162-175, 2020.

[8] T. W. Chim, S. M. Yiu, L. C. K. Hui, and V. O. Li, "VSPN: VANET-based secure and privacy-preserving navigation," IEEE Transactions on Computers, vol. 63, no. 2, pp. 510-524, 2012.

[9] P. D. Dorge, G. H. Raisoni, and M. B. Chakole, "Implementation of MIMO and AMC techniques in WiMAX network based VANET system implementation of MIMO and AMC techniques in WiMAX network based VANET system," International Journal of Information Technology and Computer Science (IJITCS), vol. 8, no. 2, pp. 60-68, 2016.

[10] V. Patel, M. Chaturvedi, and S. Srivastava, "Comparison of SUMO and SiMTraM for Indian traffic scenario 
representation," Transportation Research Procedia, vol. 17, pp. 400-407, 2016.

[11] R. Cumbal, X. Calderon, R. Hincapie, L. Urquiza, and G. Arevalo, "Performance analysis of a VANET with optimal infrastructure location in setting urban," in 2018 IEEE Colombian Conference on Communications and Computing (COLCOM), Medellin, Colombia, May 2018.

[12] F. Goudarzi, H. Asgari, and H. S. Al-Raweshidy, "Traffic-aware VANET routing for city environments-a protocol based on ant colony optimization," IEEE Systems Journal, vol. 13, no. 1, pp. 571-581, 2018.

[13] C. H. Lee, K. G. Lim, M. K. Tan, R. K. Y. Chin, and K. T. K. Teo, "A genetic algorithm for management of coding resources in VANET," in 2017 IEEE 2nd International Conference on Automatic Control and Intelligent Systems (I2CACIS), pp. 80-85, Kota Kinabalu, Malaysia, October 2017.

[14] Y. He, W. Xu, and X. Lin, "A stable routing protocol for highway mobility over vehicular ad-hoc networks," in 2015 IEEE 81st Vehicular Technology Conference (VTC Spring), pp. 1-5, Glasgow, UK, May 2015.

[15] M. H. Eiza, T. Owens, and Q. Ni, "Secure and robust multiconstrained QoS aware routing algorithm for VANETs," IEEE Transactions on Dependable and Secure Computing, vol. 13, no. 1, pp. 32-45, 2016.

[16] R. Kumar and S. K. Routray, "Ant colony based dynamic source routing for VANET," in 2016 2nd International Conference on Applied and Theoretical Computing and Communication Technology (iCATccT), pp. 279-282, Bangalore, India, 2016.

[17] A. Elbery, H. Rakha, M. Y. Elnainay, F. Filali, and W. Drira, "Eco-routing: an ant colony based approach," in International Conference on Vehicle Technology and Intelligent Transport Systems (VEHITS 2016), pp. 31-38, Rome, Italy, 2016.

[18] C. Wang, Q. Fan, X. Chen, and W. Xu, "Prediction based greedy perimeter stateless routing protocol for vehicular selforganizing network," in IOP Conference Series: Materials Science and Engineering, vol. 322, 2018no. 5, Article ID 052019.

[19] M. Jafer, M. A. Khan, S. Ur Rehman, and T. A. Zia, “Optimizing broadcasting scheme for VANETs using genetic algorithm," in 2016 IEEE 41st Conference on Local Computer Networks Workshops (LCN Workshops), pp. 222-229, Dubai, United Arab Emirates, 2016.

[20] B. Ranjan Senapati and P. Mohan Khilar, "Optimization of performance parameter for vehicular ad-hoc network (VANET) using swarm intelligence," in Nature Inspired Computing for Data Science, pp. 83-107, Springer, Cham, 2020.

[21] B. Dappuri, M. Amru, and A. M. Venkatanaga, "A stable routing algorithm based on link prediction method for clustered VANET," in Recent Trends and Advances in Artificial Intelligence and Internet of Things, pp. 85-95, Springer, 2020.

[22] H. Bello-Salau, A. M. Aibinu, Z. Wang, A. J. Onumanyi, E. N. Onwuka, and J. J. Dukiya, "An optimized routing algorithm for vehicle ad-hoc networks," Engineering Science and Technology, vol. 22, no. 3, pp. 754-766, 2019.

[23] A. M. Aibinu, H. B. Salau, N. A. Rahman, M. N. Nwohu, and C. M. Akachukwu, "A novel clustering based genetic algorithm for route optimization," Engineering Science and Technology, vol. 19, no. 4, pp. 2022-2034, 2016.

[24] S. Harifi, J. Mohammadzadeh, M. Khalilian, and S. Ebrahimnejad, "Giza pyramids construction: an ancient- inspired metaheuristic algorithm for optimization," Evolutionary Intelligence, pp. 1-19, 2020.

[25] M. L. Chiang, "Eventually byzantine agreement on CDS-based mobile ad hoc networks," Ad Hoc Networks, vol. 10, no. 3, pp. 388-400, 2012.

[26] G. D. Singh, M. Prateek, and G. H. Sastry, "A novel algorithm for efficient routing in vehicular ad hoc network using swarm intelligence optimization techniques," International Journal of Advanced Science and Technology, vol. 29, no. 7, pp. 1132$1143,2020$.

[27] M. B. Wagh and N. Gomathi, "Route discovery for vehicular ad hoc networks using modified lion algorithm," Alexandria Engineering Journal, vol. 57, no. 4, pp. 3075-3087, 2018.

[28] "Webpage, "OpenStreetMap"," https://www.openstreetmap .org/\#map=18/30.32427/78.04188.

[29] S. Jain, S. Sharma, and R. Tomar, "Integration of wit API with python coded terminal bot," in Emerging Technologies in Data Mining and Information Security, pp. 397-406, Springer, Singapore, 2019.

[30] G. D. Singh, M. Prateek, and G. H. Sastry, "Methodology to perform simulation experiments for realistic VANET scenarios using open source software tools," International Journal of Psychosocial Rehabilitation, vol. 24, no. 6, pp. 1017010175, 2020.

[31] P. Chithaluru, S. Kumar, A. Singh, A. Benslimane, and S. K. Jangir, "An energy-efficient routing scheduling based on fuzzy ranking scheme for internet of things (IoT)," IEEE Internet of Things Journal, 2021.

[32] P. P. Chatterjee, A. Deshpande, and A. Patel, "A discrete-event network simulator for internet systems," November 2020, https://www.nsnam.org/releases/ns-3-29/download/.

[33] "Dehradun clock tower|OpenStreetMap," November 2020, https://www.openstreetmap.org/search?query=dehradun clock tower\#map $=15 / 30.3321 / 78.0542$ \&layers $=\mathrm{N}$. 